\title{
Infrared thermography for detecting the deposit nature in steel water pipes
}

\author{
by A. Saifi ${ }^{*}$ H.Halloula* ,A. Elamiri* ${ }^{*}$ A.Elhassnaoui ${ }^{*}$, A.Obbadi*,Y.Errami* and S. Sahnoun* \\ * Laboratory of Electronics, Instrumentation and energetic, Faculty of Sciences, B.P 20.24000 \\ El-Jadida, Morocco. ssahnoun@gmail.com
}

\begin{abstract}
The formation of calcareous and rust layers in water pipe networks poses a significant technical problem. In this work, we studied by infrared thermography, the possibility of evaluating the deposits nature frequently found in steel water pipes such as rust and limestone. In the one-dimensional case, we calculated by simulating the thermal effusivity of deposits in steel water pipes from their surface temperatures. We have considered the case of a semi-infinite solid composed by a finite thickness layer over the top of a semi-infinite deposit. The results can be applied in the nondestructive inspection for determining the deposits nature in the water distribution pipe networks steel.
\end{abstract}

\section{Introduction}

Deposition of calcium Carbonate scale and rust in the water pipes becomes a serious problem for several industrial installations, it can lead to important technical and economic consequences: clogging and diameter reduction the water pipes, increase energy consumption [2,4]. Knowledge of the nature of these deposits allows with a non-destructive method to eliminate them or intervene by industrial processes upstream to prevent their deposit. Therefore, it is necessary to develop methods of prevention to identify the layers nature formed in the inner surface of steel water pipes.

The active technique using an infrared camera can be used for several applications such as diagnosis of fouling and clogging in pipes, detection of cavitation erosion, inspection of inner surface of side walls and cracks developed in the films which protect structures etc. ...

Based on the analysis of the one-dimensional theoretical solution of the heat conduction equation in the case of a solid compound of a finite layer associated with a semi-infinite substrate layer and subjected to a constant heat flux. We will propose a method for estimating the thermal effusivity of rust and calcium carbonate layers formed in the inner walls of steel water pipes.

\section{Contexte théorique}

For a one-dimensional semi-infinite solid compound of a finite layer thickness over a substrate semiinfinite layer thickness; when, the whole is heated by a constant heat flux (fig1)

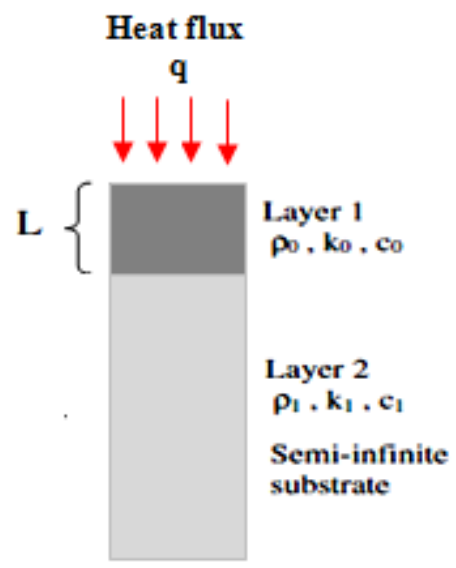

Fig.1 : Model of monodimensionnel heat transfert for a semi-finite solid 


\subsection{1/qirt.2016.111}

Osiander and al. [1] gives the theoretical surface temperature response $\mathrm{T}(\mathrm{t})$ as:

$$
\Delta T(t)=C \cdot \sqrt{t} \cdot\left[1+\sum_{n=1}^{\infty}\left[2 \cdot\left(-\Gamma^{n}\right) \cdot\left(\exp \left(\frac{-n^{2} \cdot L^{2}}{\alpha_{0} \cdot t}\right)-\frac{n \cdot L \cdot \sqrt{\pi}}{\sqrt{\alpha_{0} \cdot t}} \cdot \operatorname{erfc}\left(\frac{n \cdot L}{\sqrt{\alpha_{0} \cdot t}}\right)\right)\right]\right]
$$

Where

$$
\begin{aligned}
& C=\frac{2 . q}{k_{0}} \cdot \sqrt{\frac{\alpha_{0}}{\pi}} \\
& \alpha_{0}=\frac{k_{0}}{\rho_{0} \cdot c_{0}} \\
& \Gamma=\frac{e_{0}-e_{1}}{e_{1}+e_{0}} \\
& e=\sqrt{\rho . \kappa \cdot c} \\
& \Delta T(t)=T(t)-T\left(t_{0}\right)
\end{aligned}
$$

Where:

- $T(t)$ : The surface temperature heated at the instant $t$

- $\mathrm{T}\left(\mathrm{t}_{0}\right)$ : The surface temperature heated at the instant $\mathrm{t}_{0}$ (before the heat flux application)

- $\mathrm{q}$ : The input energy per unit area $\left(\mathrm{W} / \mathrm{m}^{2}\right)$,

- $\mathrm{L}$ : The thickness of the top layer $(\mathrm{m})$,

- $\mathrm{k}$ : The thermal conductivity(W/m.K)

- c : specific heat (J/kg.K)

- $\rho:$ density $\left(\mathrm{kg} / \mathrm{m}^{3}\right)$

- e : The thermal effusivity $\left(\mathrm{J} / \mathrm{m}^{2} . \mathrm{K}\right)$

- $\alpha$ : The thermal diffusivity $\left(\mathrm{m}^{2} / \mathrm{s}\right)$

- Г: The thermal mismatch factor.

The subscript ' 0 ' indicates specific material properties of finite thickness layer. The subscript' 1 ' indicates material properties of the semi-infinite substrate material (refer to Fig1)

For an upper layer of effusivity $e_{0}$, the knowledge of thermal mismatch factor $\Gamma$ allows to determine the effusivity $e_{1}$ of substrate layer by (4), derived from equation (7).

$$
e_{1}=e_{0}\left(\frac{1-\Gamma}{1+\Gamma}\right)
$$

- $\Gamma$ is approximately 1 if the substrate layer has a thermal effusivity $e_{1}$ much lower than the upper layer,

- $\Gamma$ is equal to 0 for two layers of the same thermal effusivity.

- $\Gamma$ is almost equal to- 1 if the second layer has a thermal effusivity higher than $\mathrm{e}_{0}$ 


\subsection{1/qirt.2016.111}

In the case of a steel pipe, we studied the thermal mismatch factor variation as a function of the temperature difference between a measurement time $t$ and the time just before applying the heat flow $t$. We studied: the effect of the measurement time $t$, the effect of heat flow $q$ and at the end we studied also the effect of the thickness $L$ of the steel layer on the surface temperature of the controlled pipe...

Our objective in this work is to determinate the thermal effusivity of the deposits formed in steel pipes. We took the case of the most frequent deposits ie rust and limestone. Note that these deposits have a much lower thermal diffusivity than that of steel (Table 1 ). In this case thermal mismatch factor $\Gamma$ value is strictly greater than 0.6 and lower than 1 ; we will take $[0.6,1]$.as variation interval of $\Gamma$.

\subsection{Effect of measurement time}

To illustrate the measurement time t. effect, we set the flux density at $6000 \mathrm{~W} / \mathrm{m}^{2}$ and the thickness of the top layer at $2 \mathrm{~mm}$. We have represented in the figure 2 the the temperature difference $\Delta \mathrm{T}(\mathrm{t})$ variation according the relation (1) as a function of the thermal mismatch factor $\Gamma$ for six different measurement time (1s, $2 \mathrm{~s}, 3 \mathrm{~s}, 5 \mathrm{~s}, 7 \mathrm{sand} 10 \mathrm{~s})$.

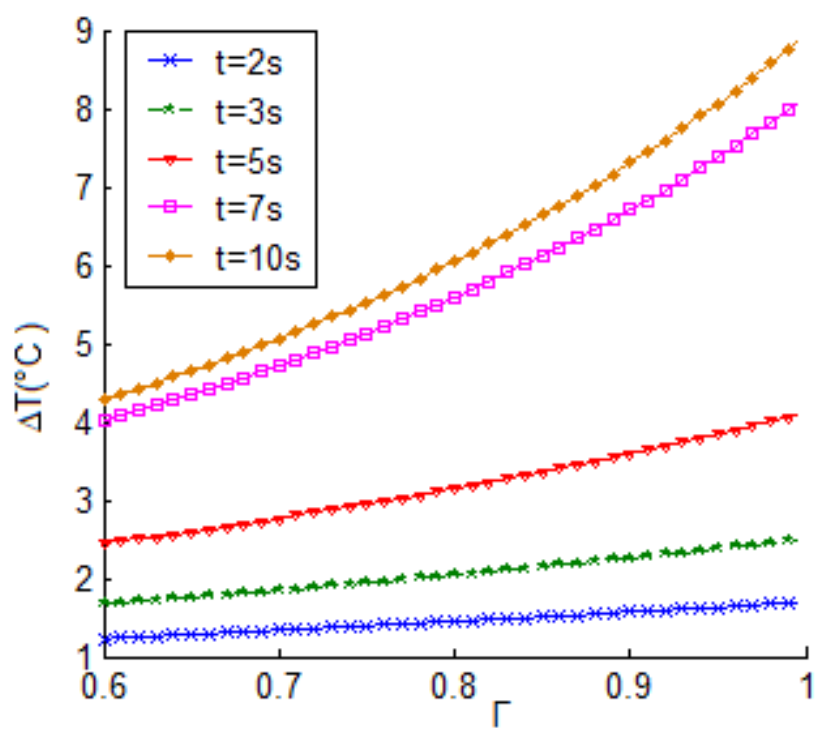

Fig.2: Temperature difference $\Delta T(t)$ as a function of the thermal mismatch factor $\Gamma$ for six different measurement time

We notice that the $\Delta \mathrm{T}$ values are low for small measurement time $1 \mathrm{~s}<\mathrm{t}<3 \mathrm{~s}$ in this interval of the thermal mismatch factor $\Gamma(0.6<\Gamma<1)$. The $\Delta T$ variations become important for measurement times above $5,5 \mathrm{~s}<\mathrm{t}$. We think that from this moment the heat flux arrive at the deposit layer after crossing the steel layer. So the choice of this time is an important parameter to determine the thermal mismatch factor between the upper layer (steel) and the deposit layer. We propose to take as time of temperature measurement $t=7 \mathrm{sec}$ because it gives a good variation of the temperature difference $\Delta \mathrm{T}$.

\subsection{The heat flux effect}

To study the effect of the heat flow density q applied to the sample surface, we fixed the thickness of the steel layer at $L=3 \mathrm{~mm}$, the measurement time is $t=7 \mathrm{~s}$. We illustrated in the figure 3 the temperature difference $\Delta T(t)$ variation as a function of the thermal mismatch factor $\Gamma$ for different values of the heat flux density $q . q=2000 \mathrm{~W} / \mathrm{m}^{2}, 4000 \mathrm{~W} / \mathrm{m}^{2}$, $6000 \mathrm{~W} / \mathrm{m}^{2}$ et $8000 \mathrm{~W} / \mathrm{m}^{2}$. 


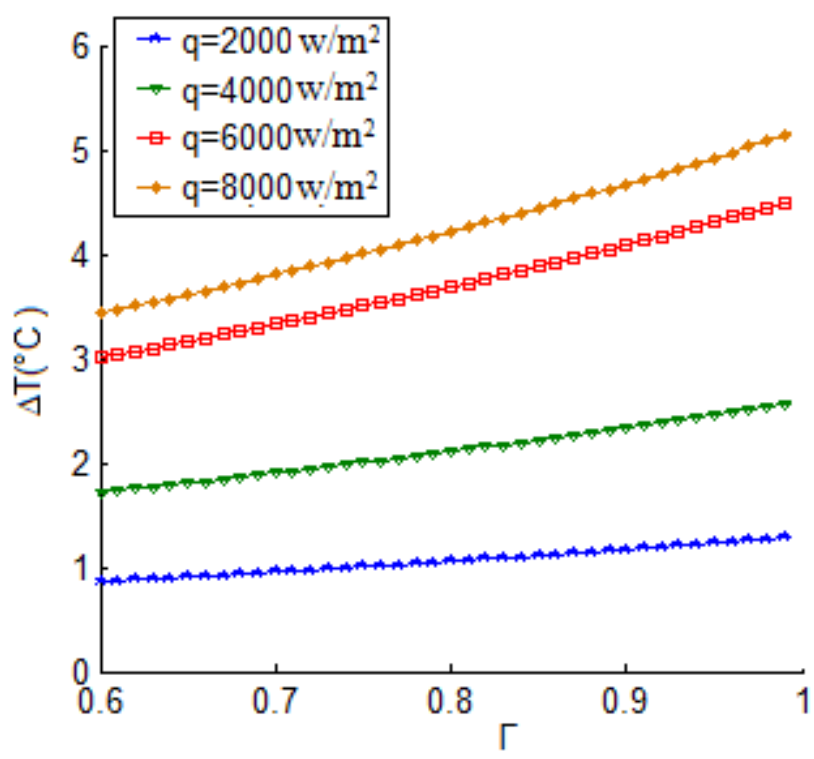

Fig.3 : Temperature difference $\Delta T(t)$ variation as a function of $t$ the thermal mismatch factor at $t=7 \mathrm{~s}$ for different values of the heat flow density $q$

Also we note that the temperature difference $\Delta T$ increase with the applied heat flux intensity. In fact the application of a heat flow with suitable intensity determines the thermal mismatch factor $\Gamma$ between the upper layer steel and the deposit layer from the temperature of the heated face measured at $t$ and to deduce the deposits effusivity.

\section{3. top layer thickness effect}

We fixed the measurement time at $t=7 \mathrm{~s}$ and the heat flux density at $q=6000 \mathrm{w} / \mathrm{m}^{2}$ to study the thickness effect $L$ of the upper steel layer. We illustrated in figure 5 the temperature difference $\Delta T(t)$ variation as a function of the thermal mismatch factor $\Gamma$ for different thickness of the upper layer $L, L$ ranged from $2 \mathrm{~mm}$ to $7 \mathrm{~mm}$.

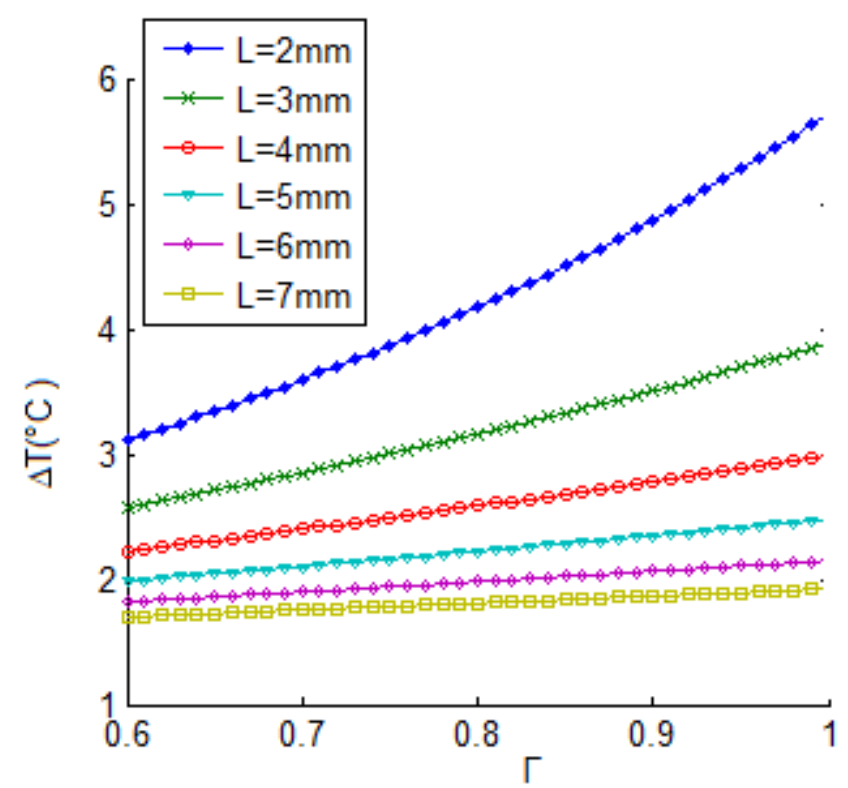

Fig.4 : Top layer thickness effect on the temperature difference $\Delta$ Tat $t=7 \mathrm{~s}$, 


\subsection{1/qirt.2016.111}

We can note that the temperature variation decreases with the thickness of the upper layer L, because it makes an obstacle to the heat diffusion. A small thickness of the upper layer allows to determine easy the thermal mismatch factor between the upper steel layer and the deposit layer.

\section{Model to estimate the thermal effusivity of deposits in steel water pipes}

After preliminary studies of some parameters on the temperature difference $\Delta T$ variation as a function of reflection coefficient between upper steel layer and a any deposit layer. Since the thickness $L$ of the tube often varies depending on the use of steel tubes; we will take it as a parameter in the proposed model, equation (8). This model determines the thermal mismatch factor $\Gamma$ from the temperature difference $\Delta T$ of the heated surface at $t=7 \mathrm{~s}$, the heat flux value is $q=6000 \mathrm{~W} / \mathrm{m}^{2}$.

$$
\Gamma=A(L)+B(L) \cdot \ln \left(T-T_{O}\right)
$$

Where

- $\Gamma:$ The thermal mismatch factor between the upper steel layer and the substrate layer.

- $\mathrm{T}$ : The measured temperature at $\mathrm{t}=7 \mathrm{~s}$.

- $T_{0}$ : The measured temperature at $t_{0}$ (before the heat flux application $q=6000 \mathrm{w} / \mathrm{m}^{2}$ )

- $L$ : The top layer thickness in $\mathrm{mm}$

The coefficients $A(L), B(L)$ are given by:

$$
\begin{aligned}
& A(L)=\left[-0.49 . L^{5}+10.53628 . L^{4}-125 \cdot 039 \cdot L^{3}+742.4 \cdot L^{2}-3656 \cdot L+3774.5\right] \cdot 10^{-4} \\
& B(L)=\left[0.724 . L^{5}-8 \cdot 9 \cdot L^{4}+87.5 \cdot L^{3}-173 \cdot 4 \cdot L^{2}+275 \cdot 26 \cdot L+1178 \cdot 8\right] \cdot 10^{-4}
\end{aligned}
$$

According to equations (7) and (8) we can calculate the thermal effusivity of deposits in steel pipes that have a thickness greater than or equal $1 \mathrm{~cm}$.

\subsection{Validation of the proposed Model}

To verify the theoretical model proposed and as we do not currently have experimental measurement means, we simulate with a computer code based on 3D finite elements, two portions of steel pipes of $0.1 \mathrm{~m}$ length and $2 \mathrm{~mm}$ thick figure 5. The inner face of the first sample contains a limestone layer while the second layer contains a rust. We applied on the outer faces of the two pipes a density of heat flux $q=6000 \mathrm{w} / \mathrm{m}^{2}$ for $t=10 \mathrm{sec}$. isolated.

The initial temperature of the two outlets pipes is $T_{0}=298.15 \mathrm{~K}$. Their lateral faces are supposed perfectly
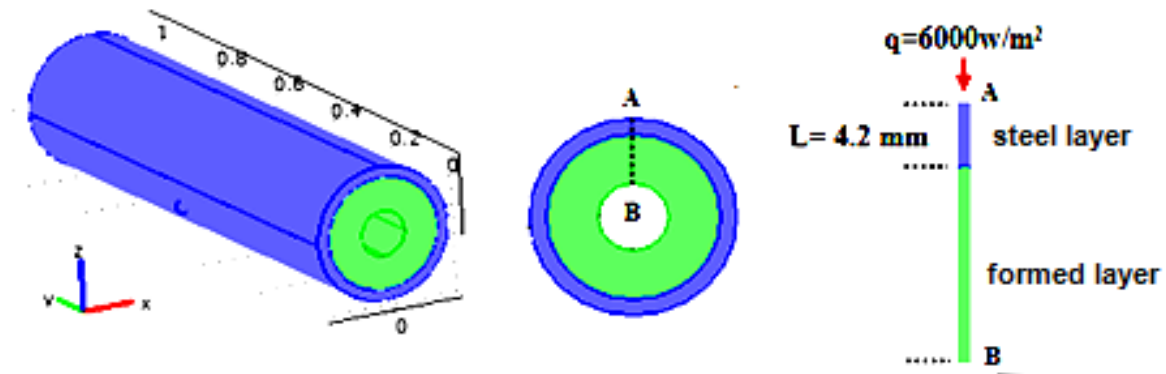

Fig.5 : simulation structure considered

The thermal properties of the considered materials in this study and the thermal mismatch factor $\Gamma$ for each layer are given in Table 1. 
10.21611/qirt.2016.111

Table 1: Thermal properties of the used materials [2,5]

\begin{tabular}{|l|l|l|l|l|l|}
\hline Materials & $\lambda\left(\mathrm{W} \cdot \mathrm{m}^{-1} \cdot \mathrm{K}^{-1}\right)$ & $\rho\left(\mathrm{Kg} \cdot \mathrm{m}^{-3}\right)$ & $\mathrm{c}\left(\mathrm{J} \cdot \mathrm{Kg}^{-1} \cdot \mathrm{K}^{-1}\right)$ & $\mathrm{e}\left(\mathrm{J} \cdot \mathrm{K}^{2} \cdot \mathrm{m}^{-2} \cdot \mathrm{s}^{1 / 2}\right)$ & $\Gamma$ \\
\hline Steel & 44.5 & 0.6 & 475 & 12881.3576 & - \\
\hline Limestone & 2.7 & 2600 & 900 & 2513.5632 & 0.6734 \\
\hline Rust & 0.6 & 1000 & 4200 & 1587.45 & 0.7805 \\
\hline
\end{tabular}

In the figure below, we plotted the evolution of the temperature of the heated face over time for both treated layers

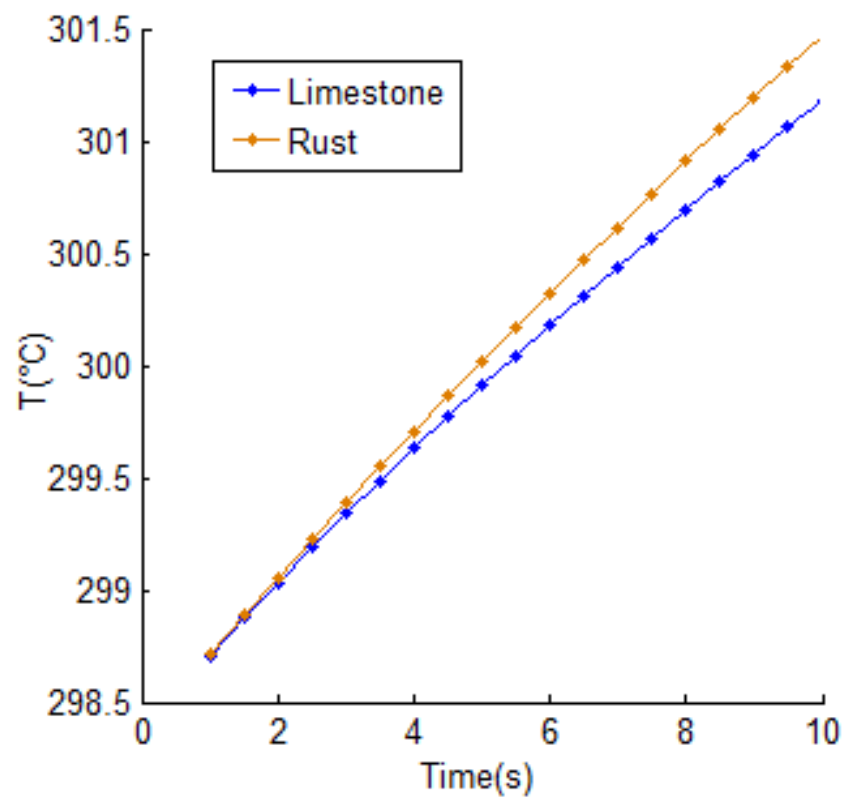

Fig.3: The temperature variation of the heated surface over time for both simulated layers limestone and rust

The determination of the deposit layers nature by calculating the thermal mismatch factor, consists in calculating the heated surface temperature $\mathrm{T}$ at $\mathrm{t}=7 \mathrm{~s}$, replace it in the proposed model and deduce the value of the deposit effusivity according to equation (7). The table shows the simulation results.

Table 2: Simulated layers thermal effusivity

\begin{tabular}{|l|c|c|c|c|}
\hline $\begin{array}{l}\text { Formed } \\
\text { Layer }\end{array}$ & $\begin{array}{c}\text { Calculated mismatch } \\
\text { factor } \Gamma\end{array}$ & $\begin{array}{c}\text { Calculated thermal effusivity } \\
\mathrm{e}\left(\mathrm{J} . \mathrm{K}^{2} \cdot \mathrm{m}^{-2} \cdot \mathrm{s}^{1 / 2}\right)\end{array}$ & $\begin{array}{c}\text { Theoretical thermal } \\
\text { effusivity }\left(\mathrm{J} . \mathrm{K}^{2} \cdot \mathrm{m}^{-2} \cdot \mathrm{s}^{1 / 2}\right)\end{array}$ & $\begin{array}{c}\text { Error } \\
\%\end{array}$ \\
\hline Limestone & 0.67337 & 2514.34999 & 2513.5632 & 0.0313 \\
\hline Rust & 0.78040 & 1588.82618 & 1587.45 & 0.0866 \\
\hline
\end{tabular}
literature.

We find a small difference between the values of effusivity provided by the model and those found in the

\section{Conclusion}

The presence of limestone or rust in water distribution networks poses a problem for private or industrial consumers. We proposed a non-destructive thermal method to determine the deposits nature in the water pipes of steel. Based on a one dimensional solution of the heat equation in a sealed steel water pipe; we studied the possibility to estimate the thermal effusivity deposit on the inner side of the controlled pipe. After a preliminary study of some parameters involved in the non-destructive thermal control; we proposed a mathematical model to determine the thermal effusivity of internal deposits from the controlled pipe surface temperature. The proposed model depend on the thickness of the inspected pipe.

To validate our model we simulated two portions of steel pipes. The first one contains a limestone layer while the second one contains a rust layer. The results show that the proposed model can be important in controlling steel water distribution networks. 


\section{REFERENCES}

[1] Jeff R. Brown a, H.R. Hamilton b,1 Quantitative infrared thermography inspection for FRP applied to concrete using single pixel analysis "journal homepage: www.elsevier.com/locate/conbuildmat"

[2] Abderrahim Saifi, Ahmed. Elhassnaoui , Assya. Elamiri Smail. Sahnoun "Characterization of scale in the steel water pipes by infrared thermography ",International Journal of Control, Energy and Electrical Engineering (CEEE), June-September (2014), Issue 3

[3] Ahmed Elhassnaoui, Abderrahim Saifi, Asseya Elamiri, and Smail Sahnoun"A simple method for determining the thermal effusivity of defects" Eur. Phys. J. Appl. Phys. (2014) 66: 20501

[4] Kang LEE "Ultrasonic Technique for Measuring the Thickness of Scale on the Inner Surfaces of Pipes“Journal of the Korean Physical Society,Vol.56, No. 2, February (2010), pp. 558_561

[5] Naouar LAAIDI1, SougratiBELATTART "The numerical modeling and the infrared thermography principle applied to the analysis of corrosion defects in oil Conduits", $11^{\text {th }}$ International Conference on Quantitative InfraRed Thermography. 